\title{
Blume-Emery-Griffiths Model in a Random Crystal Field目
}

\author{
N. S. BRANCO \\ Departamento de Física - Universidade Federal de Santa Catarina \\ 88040-900, Florianópolis, SC - Brazil; e-mail: nsbranco@fisica.ufsc.br
}

(February 3, 2018)

\begin{abstract}
We study the Blume-Emery-Griffiths model in a random crystal field in two and three dimensions, through a real-space renormalization-group approach and a mean-field approximation, respectively. According to the two-dimensional renormalization-group calculation, non-symmetry-breaking firstorder phase transitions are eliminated and symmetry-breaking discontinuous transitions are replaced by continuous ones, when disorder is introduced. On the other hand, the mean-field calculation predicts that first-order transitions are not eliminated by disorder, although some changes are introduced in the phase diagrams. We make some comments on the consequences of a degeneracy parameter, which may be relevant in martensitic transitions.
\end{abstract}

75.10.Hk; 64.60.Ak; 64.60.Kw

\section{INTRODUCTION}

The Blume-Emery-Griffiths (BEG) model is a spin1 Ising model, originally proposed to study ${ }^{3} \mathrm{He}-{ }^{4} \mathrm{He}$ mixturest. Later, it was used in the description of a variety of different physical phenomena: multicomponent fluids2, microemulsions 3 semiconductor alloyst, electronic conduction models 5 , etc. Its hamiltonian reads:

$$
\mathcal{H}=-J \sum_{<i, j>} S_{i} S_{j}-K \sum_{<i, j>} S_{i}^{2} S_{j}^{2}+\sum_{i} \Delta_{i} S_{i}^{2}
$$

where the first two sums are over all nearest-neighbor pairs on a lattice, the last one is over all sites and $S_{i}=$ $\pm 1,0 . J$ is the exchange parameter, $K$ is the biquadratic interaction and $\Delta_{i}$ is a site-dependent crystal field $\left(\Delta_{i}=\right.$ $\Delta$ for the pure model). The phase diagram of the model presents first-order and continuous phase transitipns and, for $K<0$, a rich variety of multicritical points 6 .

Nevertheless, some systems were found to be better described by a disordered BEG model, as, for instance, conventional shape memory alloys disorder materials (like aerogel, for example) 10 . From the theoretical point of view, on the other hand, it has been argued that randomness may have drastic consequences on multicritical behavior 11 12 2. In two dimensions, for instance, any infinitesimal amount of disorder supresses non-symmetry-breaking first-order phase transitions and replaces symmetry-breaking first-order phase transitions by continuous ones. The effect of disorder on three-dimensional systems is different: first-order phase transitions only disappear at a finite amount of randomnes 12. This behavior has been observed in some models 1215.

In order to study the effects of disorder on its phasediagram, we study the BEG model in a random crystal field (henceforth called RBEG model) given by the probability distribution:

$$
\mathcal{P}\left(\Delta_{i}\right)=r \delta\left(\Delta_{i}+\Delta\right)+(1-r) \delta\left(\Delta_{i}-\Delta\right)
$$

It is worthy stressing that the exact form of the disorder is not relevant to the overall qualitative consequences on the phase diagram. If randomness is chosen to be in the interactions $J$ or $K$, the qualitative effects will be the same (in what concerns first-order phase transitions). This is due to the fact that, even if the initial disorder is on the bonds (interactions $J$ or $K$ ), a scale transformation will propagate this disorder to the crystal field term, which will act just like field randomness on the coexistence boundary. Moreover, the exact form of the probability distribution is not relevant, either; we have performed calculations with other distributions and they lead to the same qualitative picture as the one found in this work.

Finally, we would like to mention that, to the best of our knowledge, the BEG model in a random crystal field has not been studied so far. Previous studies concentrated on the random Blume-Capel model $10.10 .13,6.67$, which has a simpler phase-diagram than the BEG model's.

The remainder of this paper is organized as follows. In section II we outline the mean-field approximation we use and discuss the results, in section III we present the real-space renormalization-group (RSRG) calculation (expected to hold for two-dimensional systems), and in the last section we summarize our main conclusions and comment on the influence of a degeneracy parameter $p$ on the critical behavior.

\section{MEAN-FIELD CALCULATION}

We chose an ordinary mean-field approximation to study the three-dimensional system. The procedure is rather usual and we refer the reader to Ref. 18, where a detailed discussion of the method is done. 
However, we would like to stress that the mean-field approximation we use is equivalent to a model where the interaction is of infinite-range, i.e., each spin interacts with every other spin in the system. This will have explicit consequences on the phase diagram and we will return to this point later.

Most of the information about the phase diagram is numerically calculated but some analytical results can be obtained. Among them, we can find the ground state for any values of $J, K, \Delta$ and $r$.

It is possible to show that the ground state magnetization, $m_{0}$, for $d \equiv \Delta / z J>0$, where $z$ is the coordination number of the lattice, is given by [results for $\Delta<0$ can be inferred from the mapping $(r, \Delta) \leftrightarrow(1-r,-\Delta)]$ :

$$
m_{0}=1-(1-r) \theta\left[d-(k+1)\left(\frac{1+r}{2}\right)\right]
$$

where $k \equiv K / J$ and $\theta[x]$ is the step function, such that $\theta[x]=0$ or 1 for $x<0$ or $x>0$ respectively. The ferromagnetic phase $O_{1}$ (see figures in this subsection), with $m_{0}=1$, is stable for $d \leq d_{c}=(k+1)\left(\frac{1+r}{2}\right)$, while for $d \geq d_{c}$ the ground state is such that $m_{0}=r$ (denoted $\mathrm{O}_{2}$ in our figures). Note that, except for $r=0$, the ground state is always ordered; this is a consequence of the simple mean-field approximation we used (we will return to this point below).

One can obtain the continuous transition line exactly, by expanding $\Phi_{\text {min }}$ in powers of the magnetization $m$ and taking the coefficient of $\mathrm{m}^{2}$ equal to zero:

$$
t_{c}=2\left(\frac{1-r}{2+e^{-k} e^{d / t_{c}}}+\frac{r}{2+e^{-k} e^{-d / t_{c}}}\right),
$$

where $t_{c} \equiv k_{B} T_{c} / z J$. More specifically, note that, for $d \gg 1$, the value of the critical temperature is $t_{c}=r$. So, for any value of $r \neq 0$, the critical line between the paramagnet and the $\mathrm{O}_{2}$ phases extends to $d=\infty$ (see figures in this subsection). This is not the expected behavior for a cubic lattice, for the following reason. The RBEG model for $d=\infty$ is equivalent to the site-diluted spin- $1 / 2$ Ising model, since for $\Delta=\infty$, a $+\Delta$ crystal field acting on a given site forces that site to be in the $S=0$ state (absent), while a $-\Delta$ field forces the site to be either in the state $S=1$ or in the state $S=-1$ (both represent a present site). Thus, only for high enough $r$ an infinite cluster of $S= \pm 1$ states will form and will be able to sustain order. Exactly at $r=r_{c}$, there is such an infinite cluster but its critical temperature is zero. Therefore, the critical parameter $d_{c}$ should only reach infinity for $r \geq r_{c}$. However, the simple mean-field analysis we made leads to $r_{c}=0$, since it is equivalent to model with infinite-range interactions. In some cases, 17 , more elaborated mean-field-like procedures were applied to the Blume-Capel model in a random crystal field. Briefly, the consequence of these approaches is that the transition line between $\mathrm{O}_{2}$ and $D$ phases does not extend to $d=\infty$ for all values of $r$. All other results are similar to the ones obtained with our simple mean-field approximation. We note in advance that the approach we used for the two-dimenisonal model leads to a finite value of $r_{c}$, as expected on physical grounds.

We have already pointed out that $t_{c}(\Delta=\infty)$ does not depend on $K$; this comes from the mapping between the RDBEG model and the site-diluted spin-1/2 Ising model. The $S=0$ states (absent sites) play no role in the dynamics of the model and the present sites can only be in the states $S=1$ or $S=-1$; thus, the biquadractic interaction, $K$, is irrelevant in this limit. If, for instance, the probability distribution $\mathcal{P}\left(\Delta_{i}\right)=$ $r \delta\left(\Delta_{i}\right)+(1-r) \delta\left(\Delta_{i}-\Delta\right)$ is used, the $\Delta=\infty$ limit will be equivalent to the site-diluted spin-1 Ising model; then, $t_{c}(\Delta=\infty)$ will depend on $K$. Note that the discussion in this paragraph applies to the two-dimensional case as well.

We now turn to the discussion of the $k_{B} T / z J \times \Delta / z J$ phase diagrams. In Figs. 1, 2, 3and 1 we depict sections of constant $K / J=5$, for many values of $r$. The phase diagram for $r=0$ (pure BEG model) is qualitatively the same as for $r=0.1$ (Fig. 1), except that the $\mathrm{O}_{2}$ phase is not present.

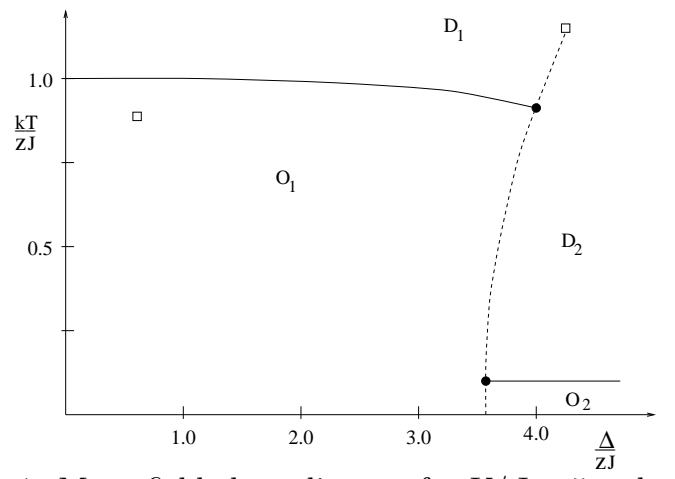

FIG. 1. Mean-field phase diagram for $K / J=5$ and $r=0.1$. Filled circles and the open square stand for critical end points and a critical point, respectively. Continuous (dashed) lines represent continuous (first-order) transitions. The phases are: ordered with $m=1\left(O_{1}\right)$, ordered with $m=r\left(O_{2}\right)$, disordered with $q>1 / 2\left(D_{1}\right)$, and disordered with $q<1 / 2\left(D_{2}\right)$.

Note that the size of the ordered phases increases with $r$. This is expected, since $r$ is the fraction of sites which feel a $-\Delta$ crystal field (we have already commented on the "tail" which separates the $O_{2}$ and $D_{2}$ phases, given by $\left.t_{c}=r\right)$. Another important feature is the presence of a first-order line between two disordered phases, for $r=0.1$ and $r=0.3$. In both of these phases $m=0$ but $q>1 / 2$ for $D_{1}$, while $q<1 / 2$ for $D_{2}$. We would like to call attention for the phase diagram for $r=0.3$ (Fig. 2); this type of diagram is not present in the Blume-Capel model. 


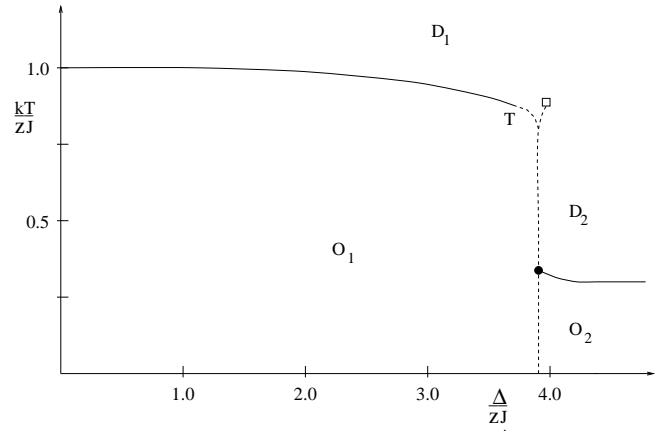

FIG. 2. Mean-field phase diagram for $K / J=5$ and $r=0.3$. Same conventions as in Fig. 1]; $T$ stands for tricritical points.

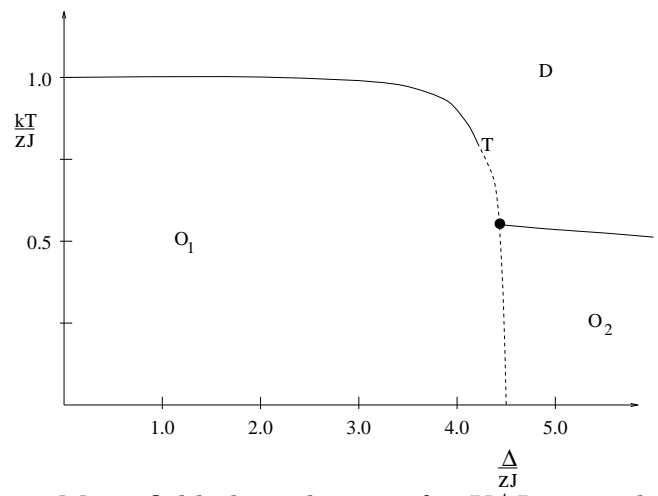

FIG. 3. Mean-field phase diagram for $K / J=5$ and $r=0.5$. Same conventions as in Fig. 2.

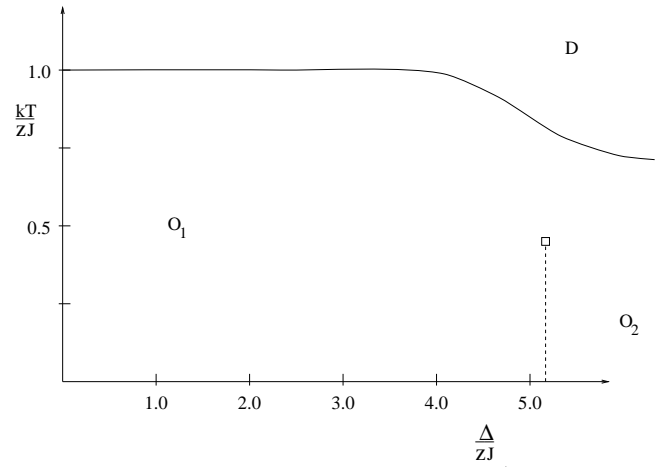

FIG. 4. Mean-field phase diagram for $K / J=5$ and $r=0.7$. Same conventions as in Fig. 2 .

The corresponding phase diagrams for $K / J=3$ show only three types of critical behavior: for $r$ near zero, they are similar to the phase diagram for $K / J=5$ and $r=0.3$ (Fig. 2); for intermediate values of $r$, the behavior is the same as for $K / J=5$ and $r=0.5$ (Fig. 3); and for $r$ close to one, the equivalence is with the diagrams for $K / J=5$ and $r=0.7$ (Fig. 价.

The Blume-Capel model $(K / J=0)$ has alraadybeen studied within mean-field approximation 9.1016 . 27 , although for different probability distributions; the results we find in this case are in qualitative agreement with those of Refs. 10 and 16 and we shall not depict all of them here. The only exception is the diagram for $r=0.1$

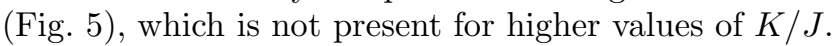

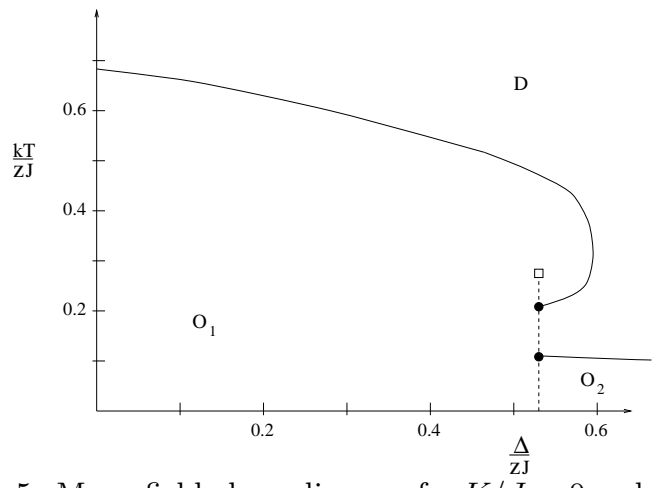

FIG. 5. Mean-field phase diagram for $K / J=0$ and $r=0.1$. Same conventions as in Fig. 2.

On general grounds, one should note that the meanfield approximation we employed suggests that the random crystal field does not destroy the first order transitions between disordered phases and between an ordered and a disordered phase. Even first order lines between ordered phases (like the one in Fig. 5) survive the introduction of randomness.

\section{TWO-DIMENSIONAL RENORMALIZATION-GROUP}

It is well known that mean-field-like approximations are not suitable to describe low-dimensional systems. We have then to resort to a different technique, in order to study the RBEG model in two dimensions. RSRG procedures, on the other hand, have been successfully applied to two-dimensional systems. Note, however, that RSRG approximations, in general, do not lead to results as precise as those obtained with Monte Carlo simulations, phenomenological renormalization or conformal invariance techniques. Nevertheless, they allow for a correct description of universality classes, order of the transitions, crossover phenomena, etc.

The procedure is the same as the one thouroughly discussed in Ref. 13. There is just one technical point we would like to stress. Although we start with a uniform distribution for $J$ and $K$, the renormalization procedure will introduce randomness in all renormalized quantities $\left(J^{\prime}, K^{\prime}\right.$ and $\left.\Delta^{\prime}\right)$. A possible approach is to follow the successive renormalized distributions of these parameters in order to study the phase diagram. We adopted an alternative way, which forces the renormalized distributions to be the same as the initial ones, but with renormalized parameters, namely, $\mathcal{P}_{a p}^{\prime}(J)=\delta\left(J-J^{\prime}\right), \mathcal{P}_{a p}^{\prime}(K)=\delta(K-$ $\left.K^{\prime}\right)$ and $\mathcal{P}_{a p}^{\prime}\left(\Delta_{i}\right)=r^{\prime} \delta\left(\Delta_{i}+\Delta^{\prime}\right)+\left(1-r^{\prime}\right) \delta\left(\Delta_{i}-\Delta^{\prime}\right)$. The values of $J^{\prime}$ and $K^{\prime}$ are obtained imposing that the first moment of the actual distributions for $J$ and $K$ and 
of $\mathcal{P}_{a p}^{\prime}(J)$ and $\mathcal{P}_{a p}^{\prime}(K)$ are equal, respectively. The values $r^{\prime}$ and $\Delta^{\prime}$ are calculated imposing that the two lowest moments of $\mathcal{P}_{a p}^{\prime}(\Delta)$ match those of the real distribution. This procedure has to be used with some care: in certain systems, where the random-field mechanism is important and the initial randomness is on the interaction ( $J$, for instance), forcing the field back into a uniform distribution leads to incorrect results. In Ref. 19, for instance, the crystal field probability distribution is maintained uniform throughout the renormalization procedure. Consequently, the critical behavior of the random model is characteristic of a high-dimensional system: the critical temperature of the tricritical point diminishes as randomness is increased but only reaches the zero temperature axis at a finite value of the disorder. As discussed in Ref. 12, the mechanism responsible for the lack of first-order phase transitions in two-dimensional random systems is the disorder in the crystal field, which is not taken into account by approximations such as the one used in Ref. 19. In the model we study in this paper, however, the disorder in the field is not approximated away by our RSRG procedure.

Our results for $K / J=2$ are presented in Fig. 6, where we depict the $k T / z J \times \Delta / z J$ phase diagram for $r=0$ (pure BEG model), $r=0.2, r=0.45$, and $r=0.5$.

Let us first comment on the pure BEG model (curve (a) of Fig. 6). As for $K / J=5$ in three dimensions, there are two types of disordered phases: both have $m=0$ but $q>1 / 2$ for phase $D_{1}$ and $q<1 / 2$ for phase $D_{2}$. The continuous line between phases $O$ and $D_{1}$ belongs to the universality class of the Ising model: this line is attracted to the Ising fixed point, $C^{*} \equiv$ $(J=0.4407, K=-0.07308, \Delta=-\infty)$. The dashed line between phases $O$ and $D_{2}$ is attracted to the fixed point $F_{1} \equiv(J=\infty, K=\infty, \Delta=2(J+K))$, which represents a first-order transition in both $m$ and $q$, i.e., the largest eigenvalue of the even and the odd sectors of the linearized RGT matrix are equal to $b^{d}$ (see Ref. 20). On the other hand, the dashed line between phases $D_{1}$ and $D_{2}$ is attracted to the fixed point $F_{2} \equiv$ $(J=0, K=\infty, \Delta=2 K+\ln 2)$; in this fixed point only the largest eigenvalue of the even sector of the linearized RGT matrix is equal to $b^{d}$; this is a sign of a discontinuity in $q$ (but not in $m$ ) when the line is crossed 20 .

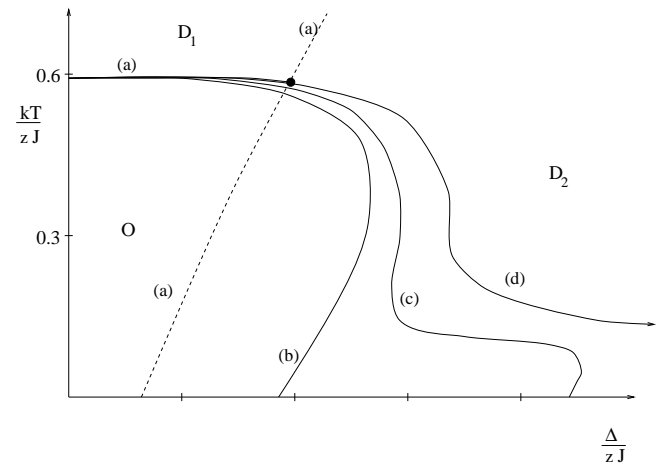

FIG. $\quad 6 . \quad$ Renormalization-group phase diagram for $K / J=2$ and (a) $r=0$, (b) $r=0.2$, (c) $r=0.45$, and (d) $r=0.5$. Filled circles stand for critical end points, $O$ for the ordered phase and $D_{1}$ and $D_{2}$ for the two disordered phases (see text). Continuous (dashed) lines represent continuous (first-order) transitions. The transition lines extend to $\Delta \rightarrow \infty$ only for $r \geq 0.5$. Note that the critical end point (filled circle) is present only for $r=0$.

In curves $(b),(c)$, and $(d)$ of Fig. 6 we depict the $k T / z J \times \Delta / z J$ phase diagram for $r \neq 0$. We note that the first-order line is either replaced by a line of continuous transitions (between $O$ and $D_{2}$ phases) or is eliminated (between $D_{1}$ and $D_{2}$ phases), for any infinitesimal amount of randomness. In fact, the first-order fixed point attractors, $F_{1}$ and $F_{2}$, are unstable along the $r$ direction. There is still a line separating the two disordered phases (not depicted in Fig. 6), $D_{1}$ and $D_{2}$, for $r \neq 0$, but this line is attracted to the $(r=1 / 2, J=0, K=0, \Delta=\infty)$ fixed point. This point represents a model with independent spins, in which no phase transition can take place. We note that our results are in accordance with general arguments on the effects of randomness on multicritical phase diagrams 12 , although, to the best of our knowledge, some features of these arguments have never been tested so far.

On the other hand, the whole line of continuous transitions for $r \neq 0$ belongs to the pure Ising model universality class, i.e., $C^{*}$ is a stable fixed-point along the $r$ direction. This is expected, since, for the hierarchical lattice used in this work, the specific heat critical exponent of the pure Ising model, $\alpha$, is negative and disorder is irrelevant, according to the Harris criterion21. For the corresponding model on a two-dimensional Bravais lattice, where $\alpha=0$, the Harris criterion is inconclusive. The accepted behavior, when disorder is present, is the following: critical exponents of the random model retain the same values as their pure conterparts byt logarithmic corrections are introduced by randomnes 22 . Experimental results also indicate the same critical expenents for pure and random two-dimensional Ising modele3.

We would like to call attention to the behavior of the critical point which separates the $O$ and $D_{2}$ phases at $T=0$. For $r<0.5$, the transition at zero temperature takes place at a finite value of $\Delta / z J$. For $r \geq 0.5$, the critical line between the ordered and the disordered phases extends to $\Delta / z J=\infty$ in the diagram. In fact, for $\Delta / z J=\infty$ the RBEG model is equivalent to the sitedilute spin-1/2 Ising model, as discussed above. Thus, only for high enough $r$ an infinite cluster of $S= \pm 1$ states will form and will be able to sustain order. There is a critical value of $r, r_{c}$, such that the critical line between the ordered and disordered phases only reaches $\Delta / z J=\infty$ for $r \geq r_{c}$. Our evaluation of $r_{c}$ is $1 / 2$, while the accepted value for the site percolation critical probability on the square lattice is $r_{c}=0.592724$. This difference is due to the small cell we use in this work; nevertheless, the correct qualitative picture is obtained, i.e., a finite value of 
$r_{c}$.

Finally, we would like to stress that there are only two types of phase diagrams for the BEG model; for high values of $K / J$ these diagrams have the same structure as for $K / J=2$. For small values of $K / J$, the structure is the same as for the Blume-Capel $(K=0)$ model. As this model has been studied elsewhere 13 , we will not discuss it here.

\section{SUMMARY}

We studied the BEG model in two and three dimensions within a RSRG framework and a mean-field approximation, respectively. The disorder is on the crystal field term, which follows a probability distribution given by: $\mathcal{P}\left(\Delta_{i}\right)=r \delta\left(\Delta_{i}+\Delta\right)+(1-r) \delta\left(\Delta_{i}-\Delta\right)$.

For the mean-field approximation (expected to represent the qualitative behavior of three-dimensional systems), the presence of randomness increases the ordered phase and brings qualitative changes to the $k T / z J \times$ $\Delta / z J$ phase diagram. More specifically, first-order transitions are present in the disordered model, but new multicritical points emerge, depending on the value of $r$.

In two dimensions, the RSRG approach we use shows that randomness has a drastic effect on critical behavior: it supresses non-symmetry-breaking first-order transitions and replaces symmetry-breaking discontinuous transitions by continuous ones. These results are in accordance with general arguments concerning the effects of quenched impurities on multicritical behavior (but, to the best of our knowledge, the disappearance of the firstorder line between disordered phases or between ordered phases has never been seen in an actual calculation). The line of continuous transition, present for the disordered $(r \neq 0)$ model, belongs to the universality class of the two-dimensional pure Ising model; this results agrees with the Harris criterion, since the specific heat critical exponent, $\alpha$, is negative for the hierarchical lattice used in this work. It has been conjectured that a new unstable critical point, at finite femperatures, might be present for the disordered system 1 ; we found no evidence of this point, for any value of $K$.

We have also studied the so-called degenerate BlumeEmery-Griffiths (DBEG) model, introduced in the study of martensitic transitions. In the DBEG model, the $S=0$ states are assumed to have a degeneracy $p$, which mimics the effects of vibrational degrees of freedom. It has been shown in Ref. 8 that the effect of increasing $p$ is to shrink the ordered phase and to increase the region where the transition is of first-order. Using the same probability distribution for the crystal field as in the RBEG model, we were able to show that the parameter $p$ may bring only quantitative changes to the phase diagrams, for any $K / J, r$, and $p$. This is expected, since the DBEG model is equivalent to the usyal $B E G$ model with all crystal fields displaced by $\ln (p) 25$. In particular, any infinitesimal amount of randomness in two dimensions destroys the first order transitions, no matter the value of $p$.

Finally, we would like to stress that our approximation does not allow for a study of the BEG model with negative $K$, where new and interesting critical behavior emergest. Work is now being made to discuss this model in the presence of a random crystal field.

\section{ACKNOWLEDGMENTS}

We would like to thank Prof. J. F. Stilck for a critical reading of the manuscript and Prof. Anna Chame for calling our attention to Ref. 8 while this work was in progress.

* Work partially supported by the Brazilian Agency FINEP.

${ }^{1}$ M. Blume, V. J. Emery, and R. B. Griffiths, Phys. Rev. A 4, 1071 (1971).

2 J. Lajzerowicz and J. Sivardière, Phys. Rev. A 11, 2079 (1975); J. Sivardière and J. Lajzerowicz, ibid. 11, 2090 (1975); 11, 2101 (1975).

${ }^{3}$ M. Schick and W.-H. Shih, Phys. Rev. B 34, 1797 (1986).

${ }^{4}$ K. E. Newman and J. D. Dow, Phys. Rev. B 27, 7495 (1983).

${ }^{5}$ S. A. Kivelson, V. J. Emery, and H. Q. Lin, Phys. Rev. B 42, 6523 (1990).

${ }^{6}$ A. N. Berker and M. Wortis, Phys. Rev. B 14, 4946 (1976).

${ }^{7}$ W. Hoston and A. N. Berker, Phys. Rev. Lett. 67, 1027 (1991).

${ }^{8}$ E. Vives, T. Castán e P.-A. Lindgård, Phys. Rev. B 53, 8915 (1996).

${ }^{9}$ C. Buzano, A. Maritan and A. Pelizzola, J. Phys.: Condens. Matter 6, 327 (1994).

${ }^{10}$ A. Maritan, M. Cieplak, M. R. Swift and F. Toigo, Phys. Rev. Lett. 69, 221 (1992).

${ }^{11}$ Y. Imry and S. K. Ma, Phys. Rev. Lett. 35, 1399 (1975).

${ }^{12}$ K. Hui and A. N. Berker, Phys. Rev. Lett. 62, 2507 (1989) or A. N. Berker, J. Appl. Phys. 70, 5941 (1991) and references therein.

13 N. S. Branco and Beatriz Boechat, Phys. Rev. B 56, 11673 (1997).

14 J. Cardy and J. L. Jacobsen, preprint cond-mat 9705038).

15 A. Falicov and A. N. Berker, Phys. Rev. Lett. 76, 4380 (1996).

${ }^{16}$ C. E. I. Carneiro, V. B. Henriques and S. R. Salinas, J. Phys. A: Math. Gen. 23, 3383, (1990), and references therein.

17 T. Kaneyoshi and J. Mielnicki, J. Phys.: Condens. Matter 2, 8773 (1990).

18 N. S. Branco and L. Bachmann, Physica A 257, 319 (1998).

${ }^{19}$ H. Dickison and J. Yeomans, J. Phys. C 16, L345 (1983). 
${ }^{20}$ M. E. Fisher and A. N. Berker, Phys. Rev. B 262507 (1982).

21 A. B. Harris, J. Phys. C 7, 1671 (1974).

${ }^{22}$ F. D. A. Aarão Reis, S. L. A. de Queiroz and R. R. dos Santos, Phys. Rev. B 54, R9616 (1996).

${ }^{23}$ I. B. Ferreira, A. R. King, V. Jaccarino, J. L. Cardy and H. J. Guggenheim, Phys. Rev. B 28, 5192 (1983); M. Hagen, R. A. Cowley, R. M. Nicklow and H. Ikeda, Phys. Rev. B 36, 401 (1987).

${ }^{24}$ D. Stauffer and A. Aharony, Introduction to Percolation Theory, $2^{\text {nd. }}$ edition (Taylor and Francis, London, 1992).

${ }^{25}$ We thank the referee for calling our attention to this mapping. 